\title{
The MMIC VCO Design for Wireless Systems at Ka-Band
}

\author{
Han-Young Lee ${ }^{\dagger}$ and Wan-Sik Kim*
}

\begin{abstract}
Reconfigurable radio technology is needed to reconstruct frequency and modem functionality, which can be different in various regions. In addition, it makes a single mobile handset capable of supporting various standards of wireless communication and thus plays a key role in mobile convergence. An MMIC VCO (voltage controlled oscillator) has been developed for high power and wide bandwidth where Clapp-Gouriet type oscillators are adapted for series feedback, and was fabricated based on 0.15um pHEMT of TRW. The MMIC VCO was connected to an aluminar substrate on the carrier for testing. This MMIC VCO module showed good performance in comparison to existing works. Furthermore, it can be potentially extended to reconfigure an MMIC VCO for wireless systems such as military applications and satellite communications.
\end{abstract}

Keywords: Clapp-Gouriet type, MMIC VCO, Wireless systems, Communication, Handset

\section{Introduction}

Reconfigurable radio technology has recently attracted much interest from researchers because it makes a mobile terminal to support various communication requirements, such as different frequencies and modems, which are needed for communication environments. High frequency, stability and tunable low noise oscillators are key components for achieving high performance, and low cost Millimeter-Wave (MMW) applications such as wireless communications and automotive radar systems [1-3]. Several oscillators operating at millimeter-wave frequencies using high electron mobility transistor (HEMT) technology have been reported [4]-[6]. The design goal of an MMIC VCO is increasing output power and bandwidth. For the purpose of this goal, the Clapp-Gouriet type is adapted for a series feedback oscillator [7]-[8] .

In this paper, the design specifications of a VCO are shown in Table 1.

It is fabricated by $0.15 \mathrm{um}$ pHEMT foundry of TRW. The developed MMIC VCO can be used for target sensing in poor environments such as wireless communication systems, radar and anti-collision radar for cars. It can also be applicable to wireless systems such as military applications and satellite communications.

Table 1. The Specifications of an MMIC VCO.

\begin{tabular}{c|c}
\hline Specifications & Design Goals \\
\hline Oscillation freq. [GHz] & 35 \\
\hline Tuning Bandwidth [GHz] & $>1.5$ \\
\hline Output Power [dBm] & $>10$ \\
\hline Phase Noise [dBc/Hz] 1 [ $1 \mathrm{MHz}$ & -100 \\
\hline Harmonics [dBc] & $<-30$ \\
\hline Bias Voltage [V] & 3 \\
\hline Tuning Voltage [V] & $3 \sim 9$ \\
\hline
\end{tabular}

$\dagger$ Corresponding Author: Division of Electronics, Information \& Communication, Daelim College, Korea. (hylee@daelim.ac.kr)

* LIG Nex1 Co., Ltd (wansik_kim@lignex1.com)

Received : June 17, 2009; Accepted : January 14, 2010

\section{Design and Fabrication}

\subsection{Design of an MMIC VCO based on a Clapp- Gouriet Oscillator}

The basic schematic of a Clapp type oscillator is shown in Fig. 1. Most RF oscillators can be explained as feedback circuits, the magnitude of loop gain to keep continuous oscillator conditions should be the unity and phase difference of the loop have to be zero. That is,

$$
\Gamma \mathrm{IN}(\mathrm{jw}) \Gamma \mathrm{L}(\mathrm{jw})=1
$$

Also, an LC tank is included to ensure a stable frequency. The basic concept of the Clapp-Gouriet oscillator consists of parallel inductance and capacitance, and the LC tank impedance is real and phase difference is zero when oscillation begins. The bias condition is $\mathrm{Vdd}=3 \mathrm{~V}(\mathrm{Id}=24.25 \mathrm{~mA})$, $\mathrm{Vgg}=-0.1 \mathrm{~V}$ for the oscillator, and output load impedance is selected for maximum output power in Fig. 2.

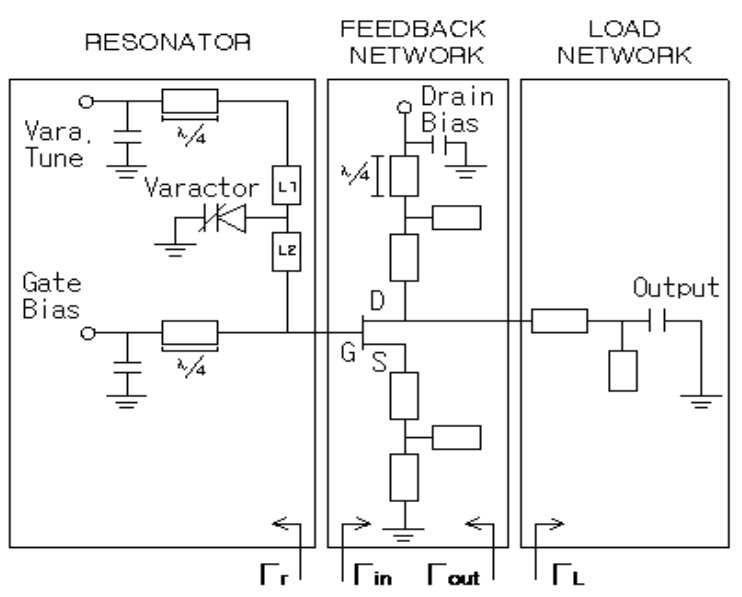

Fig. 1. MMIC VCO Circuit based on Clapp-Gouriet oscillator. 


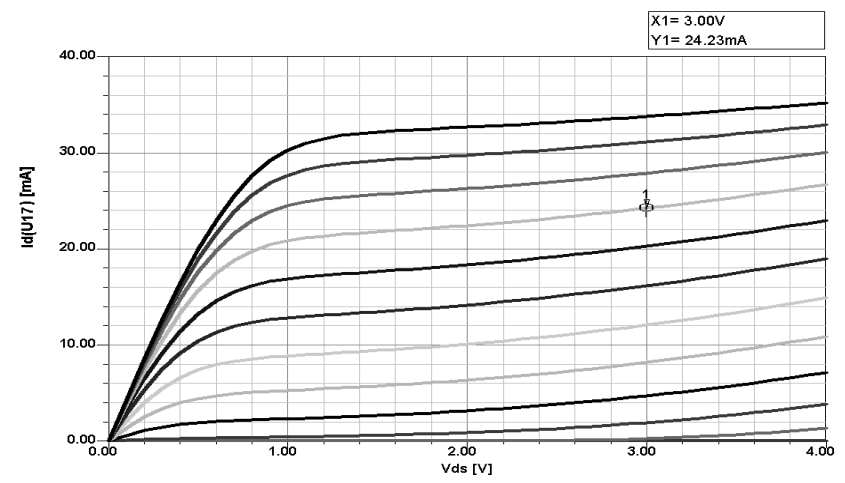

Fig. 2. Reflection coefficient of VCO.

Oscillation occurs when s11 is greater than 1 . If it appears less than 1 , a feedback inductance of $0.5 \mathrm{nH}$ is added to the open stub as the series feedback at FET Source for the purpose of $\mid \Gamma$ in $\mid>1$. Fig. 3 shows the simulation results of the reflection coefficient that the real value of S11($1.813+\mathrm{j} 0.937)$ is larger than 1 in the Smith chart [9]-[10].

The inductance value in the resonance circuit should be decided upon for the oscillation frequency of the VCO circuit. The resonance condition for oscillation is $\mathrm{XL}(\mathrm{o})=$ $\mathrm{XIN}(\mathrm{o})$ and inductance (L1) at $35 \mathrm{GHz}$ is selected as $0.367 \mathrm{nH}$ and inductance (L2) is $0.308 \mathrm{nH}$ as shown in Fig. 4.

Characteristic impedance is simulated in Fig. 5. As a result, the oscillation condition is satisfied that the real part of impedance has -439 at $35 \mathrm{GHz}$ and the imaginary part has almost zero.

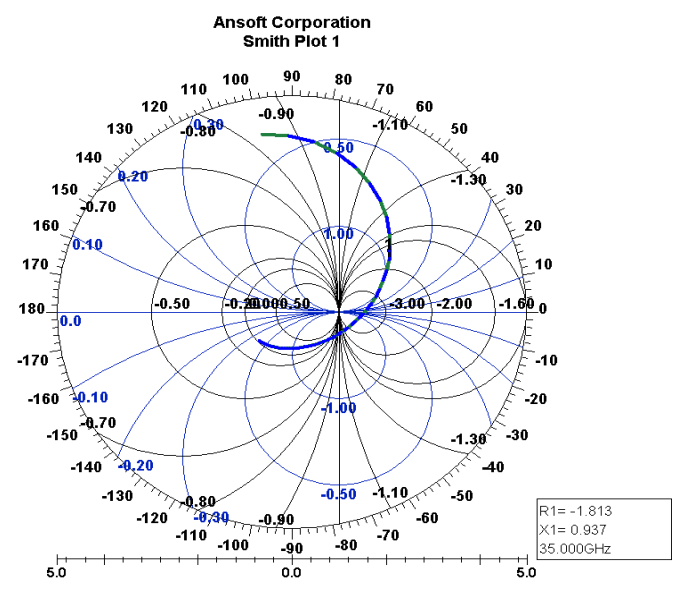

Fig. 3. Reflection coefficient of the VCO.

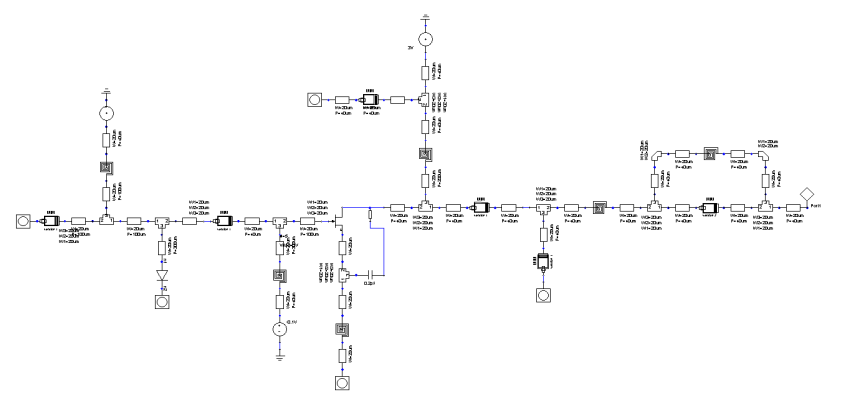

Fig. 4. Circuit of the VCO.

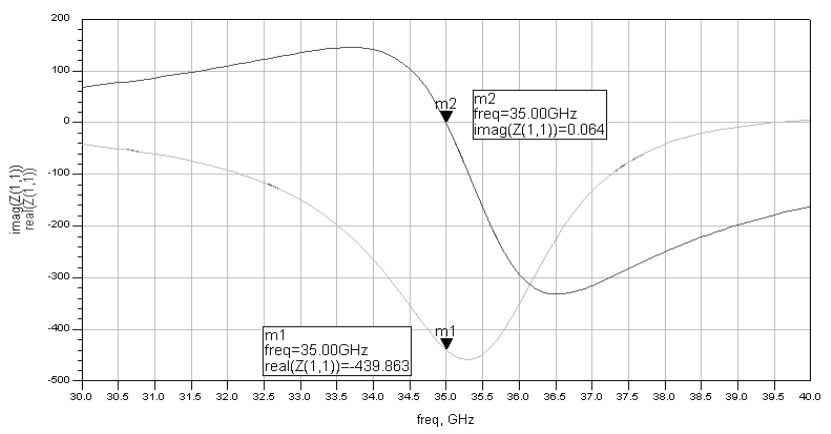

Fig. 5. Impedance of the optimized oscillator.

To achieve a good output power for the oscillation, the negative resistance looking into the collector of PHEMT devices is designed to be about three times that of the resistance looking into the output load. That is,

$$
-R_{L}(w)+j X_{L}(w)=-R_{I N}(w) / 3-j X_{I N}(w)
$$

The results of the simulation are shown in Fig. 6. The oscillation frequency of $34.73 \mathrm{GHz}$ and the output power of $10.76 \mathrm{dBm}$ when the $\mathrm{DC}$ bias condition is $\mathrm{Vdd}=3 \mathrm{~V}$ $(\mathrm{Id}=24.25 \mathrm{~mA}), \mathrm{Vgg}=-0.1 \mathrm{~V}$, and the harmonic suppression is excellent at more than $-30 \mathrm{dBc}$. The tuning range of the frequency with varactor voltage from $4 \mathrm{~V}$ to $8 \mathrm{~V}$ is shown in Fig. 7. The frequency is tuned from $34.5 \mathrm{GHz}$ to $36.5 \mathrm{GHz}$, with an approximate bandwidth of $2 \mathrm{GHz}$, and the output power is shown from $8.5 \mathrm{GHz}$ to $11.5 \mathrm{GHz}$. The simulation results are shown the phase noise of $-100 \mathrm{dBc} / \mathrm{Hz}$ at $1 \mathrm{MHz}$ offset in Fig. 8.

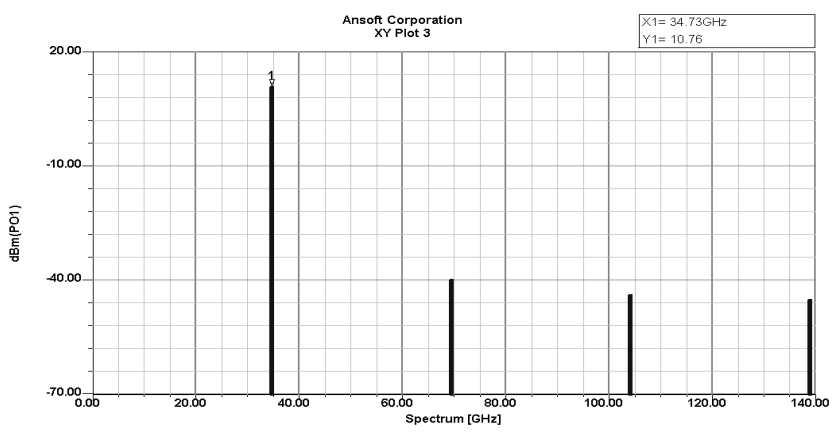

Fig. 6. Oscillation frequency characteristics of the VCO.

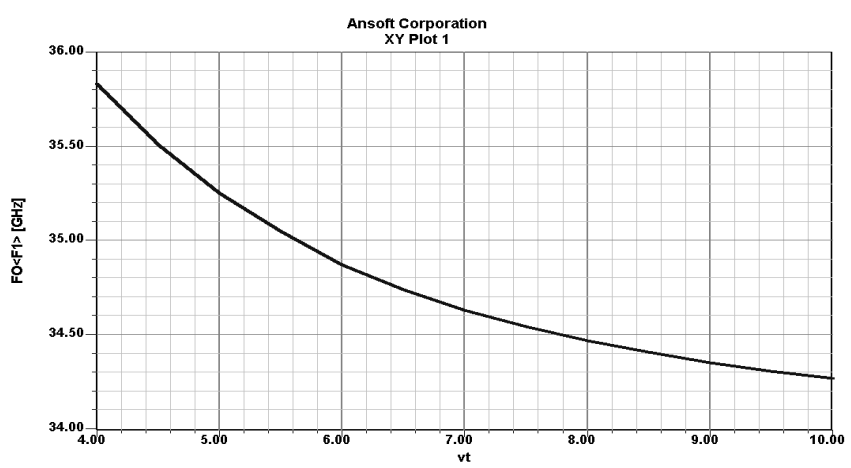

Fig. 7. The frequency response of the $35 \mathrm{GHz}$ with varactor voltage. 


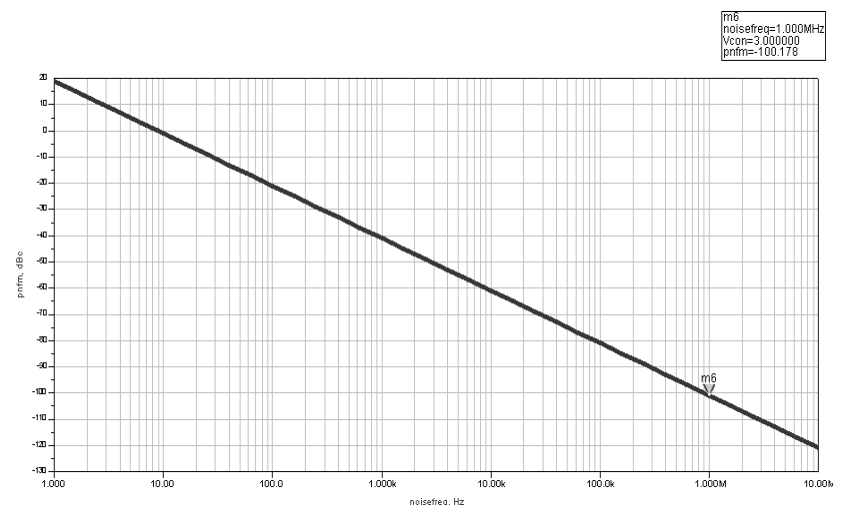

Fig. 8. Simulation results for phase noise.

The schematic of the designed circuit is shown in Fig. 9. Manufacturing used $0.15 \mathrm{um}$ pHEMT foundry of TRW.

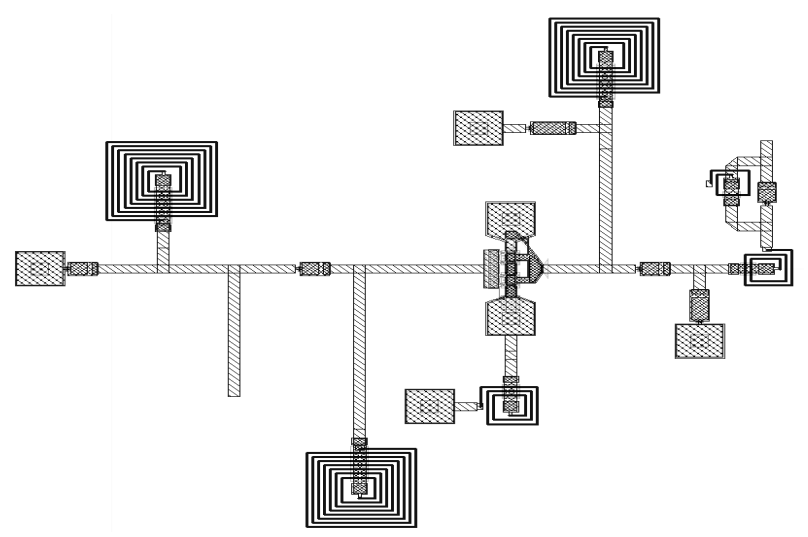

Fig. 9. Schematic of designed MMIC chip.

\section{Measured Results}

\subsection{Assembling the VCO}

Kovar is used to carrier in VCO module and the $50 \mathrm{ohm}$. Also, alumina substrate is used for the output port of the VCO MMIC. An isolator with $20 \mathrm{~dB}$ isolation characteristics is used to minimize the reflection of the VCO output power and is assembled with $0.7 \mathrm{~mm}$ diameter thickness wire bonding to minimize the transmitting power loss for each of the components shown in Fig. 10.

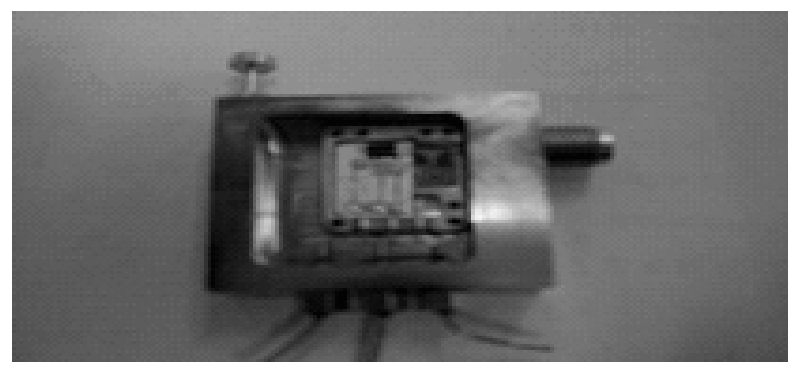

Fig. 10. Assembled VCO module (Size $=12(\mathrm{~L}) \mathrm{x} 15(\mathrm{~W}) \mathrm{x} 5$ (H) $\mathrm{mm}$ ).
A detailed illustration of the VCO module is shown in Fig. 11, and is assembled with an MMIC, alumina substrate, isolator and probe point.

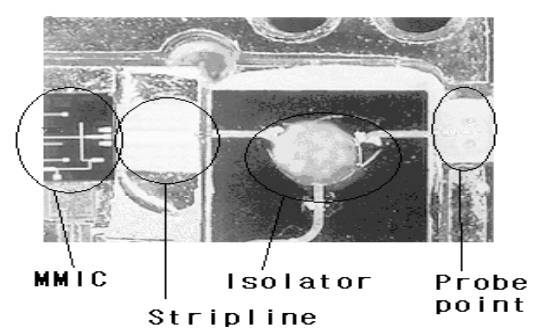

Fig. 11. Assembling the MMIC and components.

\subsection{Test Results}

The VCO test results, such as frequency and power of $11.06 \mathrm{dBm}$, are very similar to the simulation results showing a frequency of $34.73 \mathrm{GHz}$ and power of $11.06 \mathrm{dBm}$ in Fig. 12.

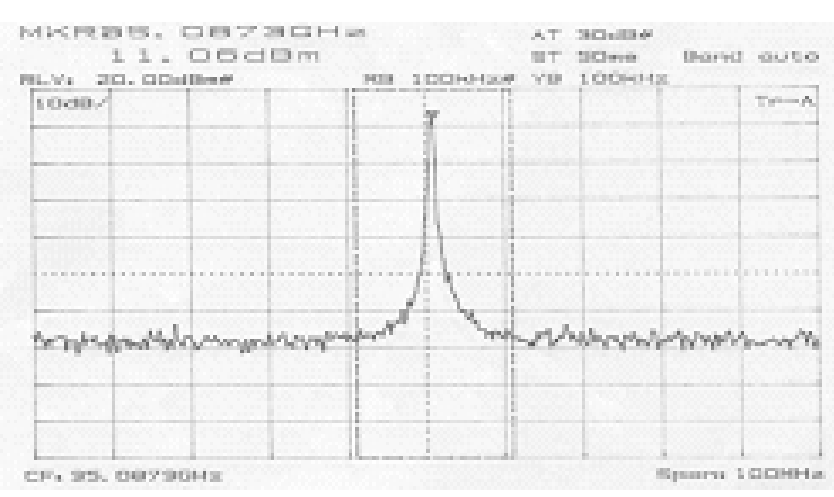

Fig. 12. Characteristics of oscillation frequency.

The non-linearity is less than $13 \%$ when the voltage range of the varactor is from $3 \mathrm{~V}$ to $8 \mathrm{~V}$, as shown in Fig. 13.

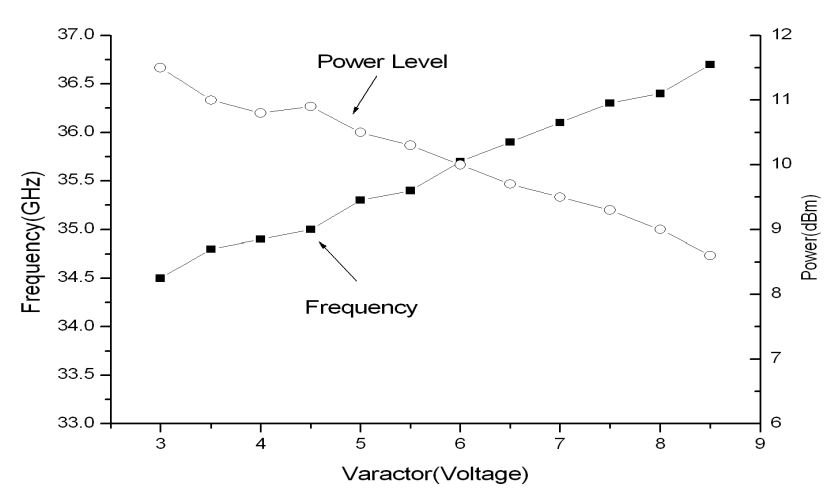

Fig. 13. Frequency and power level by tuning voltage.

The test results showed phase noise of $-90.27 \mathrm{dBc} / \mathrm{Hz}$ at $1 \mathrm{MHz}$ offset in Fig. 14, and so reached the design goal of $100 \mathrm{dBc} / \mathrm{Hz}$ at $1 \mathrm{MHz}$ offset at the bias condition. 


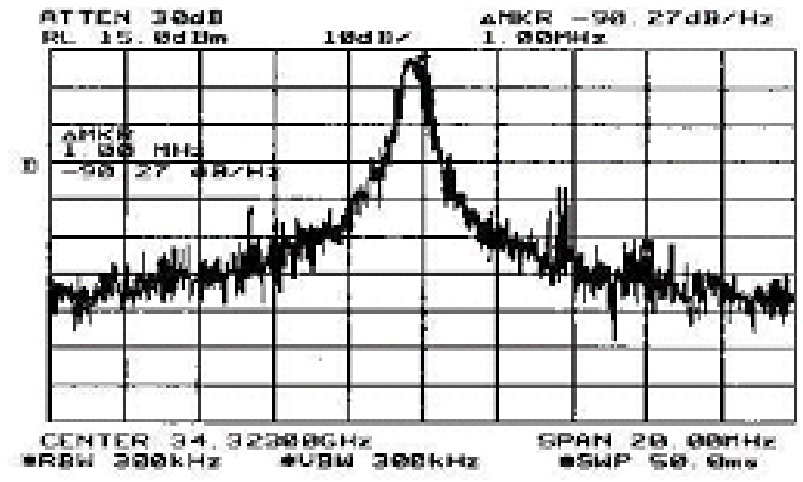

Fig. 14. Phase noise photograph of the MMIC VCO.

The VCO is assembled on a carrier fabricated with eutectic and epoxy bonding, with no carrier for biasing. Also, 0.7 mil wire bonding and 10 mil ribbon bonding are used to assemble the VCO. It requires careful fabrication skills as the tolerance error must have an influence on the system characterization [11].

Test results, such as for output power and bandwidth, show a good performance compared to the previous paper. We can see the test results in Table 2 [12]-[13] .

Table 2. Test results compared with the previous paper.

\begin{tabular}{|c|c|c|c|}
\hline Specifications & This paper & $\begin{array}{c}\text { Pervious } \\
\text { paper[12] }\end{array}$ & $\begin{array}{c}\text { Pervious } \\
\text { paper[13] }\end{array}$ \\
\hline Oscillation freq.[GHz] & 35 & 38 & 40 \\
\hline Tuning Band width[GHz] & $>2.2$ & $>0.85$ & $>2.0$ \\
\hline Output Power[dBm] & $8.5 \sim 11.5$ & $9 \sim 10.5$ & $2 \sim 6$ \\
\hline $\begin{array}{c}\text { Phase Noise[dBc/Hz] } \\
\text { @ 1MHz }\end{array}$ & -90.27 & -108 & -95 \\
\hline Harmonics[dBc] & $<-30$ & - & - \\
\hline Bias Voltage[V] & 3 & $3.5 \mathrm{~V}$ & 3.5 \\
\hline Tuning Voltage [V] & $3-9$ & $0 \sim 4$ & $0 \sim 4$ \\
\hline Device & pHEMT & InGaAs & InP HBT \\
\hline
\end{tabular}

\section{Conclusion}

In this paper, an MMIC VCO for Ka-Band is designed and assembled using using a 0.15 um pHEMT foundry of TRW. A Clapp-Gouriet type oscillator is used for series feedback in the MMIC VCO for the purpose of high output power and wide bandwidth. The VCO Module is manufactured in a small and integrated size, which is assembled on the carrier using wire and ribbon bonding with only a $0.2 \mathrm{~dB}$ insertion loss. The designed MMIC VCO was assembled for testing as a module and the test results showed a frequency of $35 \mathrm{GHz}$, a tuning bandwidth of about $2.2 \mathrm{GHz}$ and output power of $11.5 \mathrm{dBm}$. However, although it was designed as $-100 \mathrm{dBc} / \mathrm{Hz}$ at $1 \mathrm{MHz}$ offset, the phase noise was tested $-90 \mathrm{dBc} / \mathrm{Hz}$ at $1 \mathrm{MHz}$ offset. Both of the test results of output power and bandwidth show a good performance compared to previous papers.

The MMIC VCO developed can be used for target sensing in bad environments such as wireless communication systems, radar and anti-collision radars for cars. Also, it is useful for wireless systems such as military applications and satellite communication systems.

\section{References}

[1] S. A. Hovanessian, Radar System Design and Analysis, 1984.

[2] Merrill I. Skolinik, Radar Handbook (2nd Ed), McGrawHill Book Co., 1990.

[3] T. Takehana, H. Iwamoto, T. Skamoto and T. Nogami, "Millimeter-Wave radars for Automotive Use," SAE technical Paper Series Convergence '88, 1988.

[4] Y. Kwon et al., "Large signal analysis and experimental characteristics of monolithic InP-Based Wband HEMT Oscillator," 21st European Microwave Conf. Tech Dig., Stuttgart, Germany, Sept. 1991.

[5] H. Wang et al., "Monolithic W-band VCO's using pseudomorphic AlGaAs/InGaAs/GaAs HEMT's," $14^{\text {th }}$ Ann. IEEE GaAs IC Symp. Dig., Miami, FL., pp.4750, Oct., 1992.

[6] M. Funabashi et al., "A V-band AlgaAs/InGaAs hetrojunction FET MMIC dielectric oscillator," $16^{\text {th }} \mathrm{An}$ nual IEEE GaAs IC Symp. Dig., Philadelphia, PA., pp.30-33, Oct., 1994.

[7] E. Sonmez et al., "16GHz Integrated Oscillator Design with Active Elements in a Production Ready SiGe HBT MMIC Technology," EUMC, Paris, France, Oct., 2-6, 2000.

[8] Ulrich L. Rohde and David P. Newkirk, "RF/Microwave Circuit Design for Wireless Applications."

[9] Gonzales, "Microwave Transistor amplifiers Analysis and Design."

[10] Minoru Maeda, Katsuhiro Kimura, and Hiroshi Kodera, "Design and Performance of X-Band Oscillator with GaAs Schottky-Gate Field-Effect Transistor," IEEE Trans. Microwave Theory Tech., Vol.MTT-23, No.8, pp.661-667, 8, 1975.

[11] Thomas A. Midfod, "The Evolution of Package for Monolithic Microwave and Millimeter-Wave Circuits."

[12] A. Kurdoghlian et al., "38GHz Low Phase Noise CPW Monolithic VCO's Implemented in Manufacturable AlInAs/ InGaAs HBT IC technology," IEEE Trans. Microwave Theory Techniques, pp.99-102, 2000.

[13] A. Kurdoghlian et al., "40GHz Fully Integrated and Differential VCO with Wide Tuning Range in ALLNAS /INGAAS HBT," IEEE Trans. Microwave Theory Techniques, pp.129-132, 2001. 


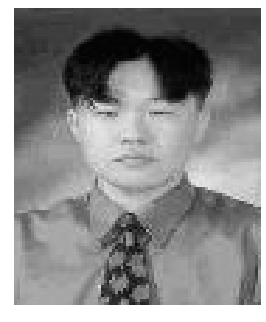

Han-Young Lee received his B.S. degree in Physics from Sejong University, his M.S. degree in Electronic Engineering from Kookmin University, and his Ph.D. degree in Electronic Information and Communication Engineering from Konkuk University in 1998, 2002 and 2005 , respectively. He is currently an Assistant at the Division of Electronics, Information and Communication, Daelim College. His main research interests are RFICs, and Microwave and Power amplifiers.

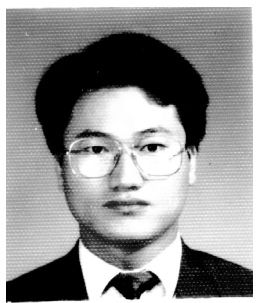

Wan-Sik Kim received his B.S., M.S. and $\mathrm{Ph} . \mathrm{D}$. degrees in Electronic Engineering from Konkuk University, Seoul, Korea in 1991, 1993 and 2005, respectively. He is currently research engineer at LIG Nex1 Co., Ltd. His main research interests are currently MMIC, Millimeter-Wave and Radar systems. 